\title{
Quando o pensamento positivo não é o bastante: 0 papel do HDML na explicação de comportamentos complexos pela RFT
}

\author{
When positive thinking is not enough: The role of the HDML framework in \\ explaining complex behavior in RFT
}

\section{Cuando el pensamiento positivo no es suficiente: el papel del HDML en la explicación de comportamientos complejos mediante RFT}

\author{
Aline Souza Simões ${ }^{1}$, Tiago Alfredo da Silva Ferreira ${ }^{1}$
}

[1] Universidade Federal da Bahia - UFBA I Título abreviado: Quando o Pensamento Positivo não é o Bastante I Endereço para correspondência: Tiago Alfredo da Silva Ferreira - Endereço de Correspondência: Instituto de Psicologia - UFBA. Rua Aristides Novis, 197, Estrada de São Lázaro. CEP 40210-730. Salvador, Bahia, Brazil Email: tiagothr@gmail.com I doi: 10.18761/PAC.2021.v12.RFT.13

Resumo: Uma tese central da Teoria das Molduras Relacionais afirma que relatos e autorrelatos não apenas descrevem o mundo, público ou privado, mas também transformam as funções deste mundo descrito. A atribuição de novos contextos verbais para ocorrência de eventos públicos e privados pode ser chamada de recontextualização verbal e é uma das principais estratégias utilizadas pela Terapia de Aceitação e Compromisso. Em uma perspectiva clínica, se considerarmos que relatos e autorrelatos promoveram funções de estímulos pouco úteis para a construção de uma vida valorosa para um sujeito, não seria, então, o caso de inserirmos outros relatos e autorrelatos? Se considerarmos autorrelatos como pensamentos, a indicação clínica seria então uma "re-programação" dos pensamentos? Para responder a tais questões, este artigo se propõe a apresentar o desenvolvimento da RFT a partir do modelo Hyper-Dimensional Multilevel (HDML) e expor a relevância deste para a compreensão do comportamento humano complexo. Inicialmente apresentaremos uma breve descrição dos avanços da RFT, desde a proposta original até o HDML. Em seguida discutiremos como a conceitualização em dimensões é essencial para compreensão da intervenção clínica baseada na RFT e concluiremos com uma reflexão sobre a relação entre a pesquisa básica na RFT e a formação de novos terapeutas.

Palavras-chave: Análise do Comportamento; Análise do Comportamento Clínica; Teoria das Molduras Relacionais; HDML. 
Abstract: A central thesis of Relational Frame Theory states that reports and self-reports not only describe the world, both public and private, but also transform the functions of the described world. Creating new verbal contexts for the occurrence of public and private events is one of the main strategies of Acceptance and Commitment Therapy. In a clinical perspective, if we consider that reports and self-report promote stimulus functions that are not very helpful for the client to build a life worth living, would the alternative be to give them new reports and self-reports? If we consider self-reports as thoughts, would the clinical intervention consist then of a type of "thought re-programming"? To answer these questions, this paper will discuss the advances in the field of RFT relating to the Hyper-Dimensional Multilevel (HDML) framework and its relevance to the understanding of complex human behavior. To start, we will describe briefly the RFT trajectory from its original proposal until HDML. Then, we will discuss how the dimensions presented in this model are essential to the understanding of clinical intervention based on RFT and we will conclude with some reflections on the relation between basic research in RFT and the development of new therapists.

Keywords: Behavior Analysis; Clinical Behavior Analysis; Relational Frame Theory; HDML.

Resumen: Una tesis central de la Teoría del Marco Relacional afirma que los relatos y los autoinformes no sólo describen el mundo, público o privado, sino que también transforman la función de este mundo descrito. La asignación de nuevos contextos verbales para la ocurrencia de eventos públicos y privados puede llamarse recontextualización verbal y es una de las principales estrategias utilizadas por la Terapia de Aceptación y Compromiso. Desde una perspectiva clínica, si consideramos que los informes y autoinformes promovieron funciones de estímulo poco útiles para la construcción de una vida valiosa para un sujeto, ¿no sería entonces el caso de insertar otros informes y autoinformes? Si consideramos los autoinformes como pensamientos, ¿̇la indicación clínica sería una "reprogramación" de los pensamientos? Para responder a estas preguntas, este artículo propone presentar el desarrollo de la RFT a partir del modelo multinivel hiperdimensional (HDML) y exponer su relevancia para la comprensión de la conducta humana compleja. Inicialmente presentaremos una breve descripción de los avances del RFT, desde la propuesta original hasta el HDML. A continuación, discutiremos cómo la conceptualización en dimensiones es esencial para entender la intervención clínica basada en la RFT, y concluiremos con una reflexión sobre la relación entre la investigación básica en RFT y la formación de nuevos terapeutas.

Palabras clave: Análisis de la Conducta; Análisis de la Conducta Clínica; Teoría de Los Marcos Relacionales; HDML. 
Uma tese central da Teoria das Molduras Relacionais (do inglês, Relational Frame Theory, RFT) afirma que relatos e autorrelatos não apenas descrevem o mundo, público ou privado, mas também transformam as funções deste mundo descrito. Isto implica dizer que o controle exercido pelo ambiente (público ou privado) é alterado a partir dos relatos, influenciando o comportar-se na direção das relações verbais estabelecidas a partir do controle verbal. Neste sentido, a RFT considera que a linguagem enquanto prática cultural possui lugar privilegiado na compreensão da subjetividade humana e na maneira como indivíduos se relacionam com sua própria subjetividade (Barnes-Holmes et al., 2018).

Por exemplo, quando um indivíduo recebe um diagnóstico psicopatológico de depressão, a sua relação com seus sentimentos e pensamentos é potencialmente alterada favorecendo, em geral, o refinamento de repertórios de esquiva destes mesmos sentimentos e pensamentos. Esta esquiva da própria experiência (i.e., esquiva experiencial) é tipificada em situações nas quais pessoas utilizam estratégias diversas para reduzir a frequência e magnitude de seus eventos privados ${ }^{1}$. Tais estratégias envolvem desde a utilização de medicações, até mesmo o isolamento de contextos evocadores de tais eventos. Um problema se instala quando tais contextos também são essenciais para a vida que o sujeito considera como significativa (e.g., relacionamentos com pessoas significativas, ambiente de trabalho, etc).

Considerando o exemplo da depressão, um desafio das terapias que se fundamentam na RFT é o de transformar a relação do sujeito com seus próprios eventos privados com vistas para o desenvolvimento de um repertório valoroso. Valores, em uma perspectiva da RFT são qualidades estáveis e abrangentes do comportar-se, descritas pelo sujeito em regras do tipo augmental ${ }^{2}$ que estabelecem

1 Para uma revisão de pesquisas acerca do papel da esquiva experiencial em categorias psicopatológicas, ver Chawla e ostafin (2007).

2 Augmentals podem ser definidos como operações motivadoras verbais que aumentam ou diminuem a efetividade de estímulos como reforçadores ou punidores (Hayes, BarnesHolmes \& Roche, 2001). funções reforçadoras para o próprio comportar-se (Ferreira et al., 2020). Uma vez que comportamentos com tais qualidades frequentemente estão relacionados a sentimentos que adquiriram função aversiva a partir de contextos verbais (e.g., o diagnóstico psicopatológico), a terapia precisa promover contingências verbais que possam mobilizar novas formas para o sujeito relacionar-se com seus próprios sentimentos e pensamentos.

A atribuição de novos contextos verbais para ocorrência de eventos públicos e privados pode ser chamada de recontextualização verbal e é uma das principais estratégias utilizadas pela Terapia de Aceitação e Compromisso (ACT) desde o seu surgimento na década de 80 (Hayes, 1987). A ACT é um dos principais modelos clínicos que se fundamentam na RFT e considera que a utilização da linguagem pelo terapeuta é a maneira fundamental de mobilizar a mudança clínica (Hayes, Strosahl \& Wilson, 2012). No entanto, caso a proposta da RFT seja caracterizada apenas pela assertiva de que novos contextos verbais alteram as funções de estímulos, a tarefa da terapia incorre em uma imagem excessivamente simplista: considerando que relatos e autorrelatos promoveram funções de estímulos pouco úteis para a construção de uma vida valorosa para este sujeito, não seria, então, o caso de inserirmos outros relatos e autorrelatos? Se considerarmos autorrelatos como pensamentos, a indicação clínica seria então uma certa "re-programação" dos pensamentos, substituindo-os por outros mais adaptativos?

No entanto, a experiência cotidiana não sustenta esta simplificação. Qualquer pessoa já pode ter experienciado um dia inteiro de elogios (relatos) no trabalho, que a fizeram pensar sobre si mesma de uma maneira gratificante (autorrelatos), e percebeu que bastou apenas uma única crítica ao final do dia para que sentimentos difíceis (e.g., vergonha, culpa, raiva) ocupassem o protagonismo da experiência privada. Por que, então, relatos elogiosos ou pensamentos positivos não seriam o bastante? Há no ser humano uma facilidade maior para o sofrimento do que para a felicidade? Ou precisaríamos recorrer ao mentalismo inerente em "confiamos mais em alguns relatos do que em outros" como causa destes fenômenos? Como a RFT explica o fato de que alguns estímulos verbais parecem ter um poder maior do que outros? 
A resposta mais coerente é a de que a RFT não contém tal explicação, ao menos em sua proposta original. Com a expressão "proposta original", nos referimos ao conteúdo exposto no livro seminal da teoria, publicado em 2001 (Hayes, BarnesHolmes \& Roche, 2001). Não obstante, os anos subsequentes têm sido prolíficos em avanços na RFT que a tornam mais adequada à explicação do comportamento humano complexo, com uma interface mais consistente com os fenômenos clínicos. Este artigo possui por objetivo apresentar o desenvolvimento recente da RFT a partir do modelo Hyper-Dimensional Multilevel (HDML) e expor a relevância desta nova proposta para a compreensão do comportamento humano complexo, em especial quando relacionado com fenômenos clínicos. Para tanto, apresentamos uma breve descrição dos avanços da RFT, desde a proposta original até o HDML. Em seguida faremos uma exposição de como a conceitualização em dimensões é essencial para compreensão da intervenção clínica com base na RFT e concluiremos com uma reflexão sobre a relação entre a pesquisa básica na RFT e a formação de novos terapeutas.

\section{Um Breve Histórico da RFT}

Para começar a compreender a RFT, é importante que se tenha uma noção do seu conceito base: o responder relacional arbitrariamente aplicável (RRAA). A partir desse conceito, pode-se trabalhar com respostas a relações entre estímulos, em detrimento de respostas a um estímulo particular. Enquanto bebês humanos e não-humanos podem ser ensinados a discriminar entre estímulos por propriedades formais (e.g., escolher o maior entre dois objetos), os humanos, através do aprendizado da linguagem, podem discriminar entre estímulos por propriedades contextuais ou sociais arbitrárias (Hayes, et al., 2001).

O RRAA é caracterizado a partir de três propriedades: implicação mútua (se A está, de alguma forma, relacionado a $B$, então pode-se dizer seguramente que $B$ também está relacionado a $A$ ), implicação combinatória (se $\mathrm{A}$ está relacionado a $\mathrm{B}$, e $\mathrm{B}$ está relacionado a $\mathrm{C}$, pode-se entender que $\mathrm{A}$ está relacionado a C) e transformação de funções de estímulos (a modificação das funções de uma das partes da relação acarretará em transformações das funções das outras partes a ela relacionadas) (Hayes et. al., 2001). Essas propriedades possuem especial importância para a compreensão dos fenômenos clínicos.

Para ilustrar as diferentes propriedades, pensemos que Pedro é um estudante de psicologia que acabou de ser aprovado em uma seleção de estágio para $\mathrm{RH}$ de uma empresa. Ele está conversando com Clara, que já estagia nesta empresa há algum tempo, e Alice, que foi contratada após se formar, alguns meses antes. Pedro fala sobre como se sente ansioso com o trabalho e as demandas da empresa, sendo o seu primeiro estágio. Clara então fala que, se ele está se sentindo ansioso agora, vai ficar muito pior depois, quando começar a se ocupar mais e as exigências aumentarem. Alice, por sua vez, destaca que ser estagiária "não foi nada". Continua seu relato afirmando que o difícil mesmo é ser contratada, já que precisa trabalhar mais horas e é bem mais cobrada. A partir desse exemplo, podemos derivar a compreensão das propriedades do RRAA. Se ser estagiário depois de um tempo é mais difícil que ao acabar de ser selecionado, podemos entender, a partir da propriedade de implicação mútua, que ser recém-selecionado é melhor que ser estagiário há mais tempo. Além disso, se estagiar há um tempo é pior que ser recém-selecionado, e ser contratado é pior que ser um estagiário experiente, pode-se compreender que ser contratado é bem pior que ser recém-selecionado - embora Alice nunca tenha falado isso diretamente (implicação combinatória). Dessa maneira, se Pedro se sentia ansioso por ter sido recém-selecionado para a vaga de estágio, a partir da transformação de funções de estímulos, podemos supor que ele ficou ainda mais ansioso ao pensar nas perspectivas futuras de contratação, ainda que não tenha tido qualquer experiência direta com o trabalho ou tenha sequer pensado se gostaria de ser contratado.

A partir do momento em que estímulos estão verbalmente relacionados, pode-se dizer que eles fazem parte de uma moldura relacional (do inglês, "relational frame"), termo que se refere a uma classe particular de RRAA, controlada pelo contexto específico que evoca a sua ocorrência (Perez et al., 2013). Existem diversos tipos de molduras relacionais, que indicam o tipo de relação entre os estímu- 
los envolvidos: coordenação, oposição, distinção, comparação, hierarquia, moldura temporal, espacial e dêitica ${ }^{3}$. A moldura relacional é estabelecida a partir de um histórico de reforçamento de múltiplos exemplares e, uma vez estabelecida, constrói-se como um operante generalizado, podendo ser aplicada junto a qualquer conjunto de estímulos a partir da utilização de dicas contextuais (BarnesHolmes et al., 2020). Assim, no desenvolvimento humano típico, uma vez que a criança vai aprendendo as suas primeiras palavras a partir da relação de coordenação (e.g., na presença do pai, um adulto nomeia "olha o papai"), a utilização da dica contextual "é igual a" infinitas relações entre estímulos.

Dicas contextuais são estímulos que exercem controle sobre quais funções comportamentais são evocadas a partir de um determinado RRAA. Ser selecionado para uma vaga de estágio, por exemplo, poderia evocar respostas diferentes se perguntássemos a Pedro quão difícil foi o processo seletivo ou quão importante esse estágio é para a sua formação no curso. Aqui, dois tipos de dicas contextuais estão envolvidos: Crel (i.e., dica ou contexto relacional) é o nome dado ao estímulo que evoca o tipo de relação envolvida, enquanto Cfunc (i.e., dica ou contexto funcional) se refere ao estímulo que exerce controle sobre quais funções comportamentais específicas estão sendo evocadas por aquele exemplar específico de RRAA. Assim, quando Clara diz a Pedro que estar estagiando há um tempo é mais difícil e demandante do que ser um estagiário recém-selecionado, podemos inferir que o Crel indica uma relação de comparação (i.e., a estrutura "mais que" indica o tipo de relação estabelecida), enquanto o Cfunc indica a característica do estímulo "estagiar há um tempo" referido nessa relação, estabelecendo as funções que serão evocadas pelos

3 Os tipos de molduras ficarão mais claros ao longo do texto, porém, para uma visão mais completa sobre o tema e os estudos experimentais envolvidos no estabelecimento das categorias, ver Hughes e Barnes-Holmes (2016).

4 “É igual a" é um dos tipos possíveis de dicas contextuais para estabelecer relações de coordenação. Essa relação pode ser estabelecida com inúmeras possibilidades de dicas, como no exemplo "essa é a mamãe" ou mesmo se o adulto somente apontar para a mãe e disser "mamãe". estímulos através da relação de comparação (i.e., a palavra "difícil", por exemplo, indica que os dois estímulos estão sendo comparados em termos de sua facilidade ou dificuldade de trabalho e a comparação nesses termos terá impacto para a função que os estímulos exercerão sobre o comportamento de Pedro).

\section{Desenvolvimentos Posteriores: avancos conceituais e a proposta do MDML}

Em 2017, Barnes-Holmes e colaboradores publicaram um artigo no JCBS (Journal of Contextual Behavioral Science) com a proposta de uma estrutura de análise complementar de pesquisa em RFT, chamada multi-dimensional multi-level (MDML), na qual descrevem especificações que podem enriquecer a contribuição dos princípios da RFT à prática clínica. Essa estrutura de análise propõe caracterizar o RRAA em quatro dimensões (coerência, complexidade, derivação e flexibilidade) e cinco níveis (implicação mútua, molduras relacionais, redes relacionais, relações entre relações e relações entre redes relacionais). A ideia é que, ao descrever essas subcategorias, possamos observar sutilezas e características do RRAA "em movimento", o que não seria possível se apenas o tratássemos como uma unidade geral.

Ao falar dos diferentes níveis, a distinção essencial se dá em termos de complexidade das relações. A relação de coordenação entre o animal gato e a palavra falada "gato" seria um exemplo de implicação mútua, uma relação simples entre dois estímulos. Na medida em que se aumenta o número de estímulos e a complexidade das relações, é possível pensar nos outros níveis, passando da relação simples entre animal e palavra até uma elaboração complexa de um cliente sobre o que o leva a se comportar de uma determinada maneira (exemplo: o cliente pode se descrever "desconfiado como um gato"). Quando o foco é estabelecido na experiência clínica, é natural que se entenda que enfatizamos as relações entre relações e as relações entre redes relacionais (i.e., níveis mais complexos), uma vez que mesmo uma simples afirmação como, por exemplo, "eu sou uma pessoa difícil" já implica em uma série 
de relações envolvendo cada um de seus estímulos componentes - só o estímulo "eu”, por exemplo, já pode estar envolvido em inúmeras outras relações.

A distinção entre níveis pode direcionar o foco clínico para elementos que ainda necessitam de clarificação em uma conceitualização de caso. Assim, podemos classificar "eu sou uma pessoa difícil" como uma implicação mútua estabelecendo uma simples relação de coordenação entre "eu" e "difícil", como uma forma de observar que ainda não temos informações suficientes sobre o cliente para compreender outros tipos de relações que possam estar envolvidas. Por exemplo: podemos considerar diversas relações sobre Catarina, uma cliente fictícia, como implicação mútua - ela é uma mulher, é homossexual, é filha, dentre outros. Quando obtemos essas informações sobre Catarina, as consideramos como implicação mútua, porque a relação de coordenação entre o "eu" e esses estímulos é tudo que sabemos. Porém, à medida que vamos sabendo mais, mudamos a nossa interpretação sobre o nível. Assim, na história de Catarina, o estímulo "mulher" pode ser entendido como alguém "delicado" e "subserviente", e diversas relações são derivadas disso, como "para ser bem vista, é importante não falar muito palavrão" (moldura condicional) e "é melhor ser uma mulher passiva do que uma mulher agressiva" (moldura de comparação). A partir disso, a relação de coordenação entre "eu" - agora relacionado a muitas características de Catarina - e "mulher" - que está vinculado a inúmeras outras relações - já pode ser compreendida como uma relação entre molduras relacionais. Se aumentarmos a complexidade e passarmos a compreender ainda mais os significados de cada elemento dessas relações, podemos falar de redes relacionais. Dessa maneira, a classificação através do MDML em termos de níveis pode ser útil para mapear o nível de profundidade da nossa compreensão sobre a função que a narrativa trazida pelo cliente exerce em sua vida. Um outro ponto importante de ser mencionado diz respeito à possibilidade de avaliar a força do RRAA. Até então, tínhamos ferramentas que permitiam avaliar se um RRAA existia ou não no repertório de um sujeito, enquanto o MDML traz a possibilidade de avaliar a força daquela resposta, uma vez que esteja presente.
Quando falamos em dimensões, coerência diz respeito ao quanto uma instância de responder relacional produz uma consequência previsível, uma vez que se sobrepõe funcionalmente a padrões anteriores de respostas que foram reforçadas (ou, ao menos, não punidas) pela comunidade verbal. Em termos mais simples, o quanto aquela relação é consistente com outros padrões de resposta na vida do sujeito. Quanto mais pervasiva e presente ela for, maior a sua coerência. Complexidade diz respeito ao nível de detalhamento daquela relação e à quantidade de tipos de relações envolvidas. Uma implicação mútua de comparação, por exemplo, é mais complexa que uma de coordenação, uma vez que a primeira envolve dois tipos de relações (A é melhor que $B, \log$ o é pior que $A$ ), enquanto a segunda envolve apenas um (A é igual a B, logo B é igual a A).

Além disso, quando falamos em um número maior de relações (i.e., redes relacionais), quanto mais estímulos estão envolvidos em uma rede relacional, maior a sua complexidade. Derivação diz respeito ao quão nova ou "praticada" aquela relação é. Em outras palavras, podemos dizer que o nível de derivação varia se a resposta verbal foi recentemente aprendida ou se já foi emitida outras vezes no histórico de interações verbais daquele indivíduo. Quando um sujeito aprende algo verbalmente, uma resposta é derivada. Na medida em que ele volta a emitir essa resposta, ela passa a sofrer consequências naturais do contexto, diminuindo então o seu nível de derivação. Assim, a primeira vez em que um estudante de análise do comportamento ouve os termos "reforçamento positivo" e "reforçamento negativo", essas relações possuem um alto nível de derivação. Na medida em que ele progressivamente desenvolve mais contato com os termos, o nível de derivação diminui até o ponto em que a resposta se torna quase automática devido às inúmeras derivações. Por último, flexibilidade diz respeito à facilidade com a qual a resposta em questão pode ser modificada a partir do seu contexto. Respostas mais rígidas e difíceis de mudar teriam um menor nível de flexibilidade (Barnes-Holmes, Barnes-Holmes, McEnteggart \& Harte, 2021).

Um ponto importante de ser reconhecido aqui é que a flexibilidade enquanto dimensão do responder relacional não deve ser confundida com 
flexibilidade psicológica5. Ao contrário desta última, a primeira não é sempre desejada. Assim, se um indivíduo está em uma situação de risco à sua vida, por exemplo, é importante que a resposta seja rígida e automática de forma a garantir o bem-estar e a sobrevivência.

\section{Limitações do MDML e Atualizações}

A interação de cada nível com cada dimensão gera 20 unidades de análise do RRAA que possibilitam o estudo desse comportamento em suas diversas nuances ${ }^{6}$. As unidades de análise refletem dinâmicas que já vinham sendo exploradas pela pesquisa básica de maneira implícita. É a partir justamente de avanços experimentais, mais especificamente através de estudos com o Implicit Relational Assessment Procedure (IRAP), que algumas limitações de foco do MDML são explicitadas e um modelo experimental é desenvolvido: o Differential Arbitrarily Applicable Relational Responding Effects (DAARRE). Enquanto campo, a RFT se desenvolveu principalmente a partir da sua pesquisa básica, sempre trazendo importantes implicações para a compreensão do trabalho clínico ${ }^{7}$.

A partir do desenvolvimento do modelo DAARRE, algumas limitações de foco do MDML são explicitadas. Até aqui, o foco adotado estava no tipo de relações envolvidas (i.e., coordenação, distinção, comparação, etc). Se resgatarmos os tipos de dicas contextuais, podemos dizer que nosso foco até então está na Crel. Porém, os avanços experimentais resgatam a importância de ampliarmos o foco também para a Cfunc, isto é, para as funções

5 Para maior compreensão acerca do conceito de "flexibilidade psicológica”, ver Hayes, Strosahl e Wilson (2012).

6 Para maior compreensão sobre a utilização das referidas unidades de análise na pesquisa básica, conferir BarnesHolmes, Finn, McEnteggart e Barnes-Holmes (2018).

7 Para o propósito do presente capítulo, porém, o foco será mantido nas implicações clínicas. Caso o leitor queira se aprofundar a respeito dos avanços que embasam esse entendimento, existe uma vasta literatura a ser consultada. Conferir Barnes-Holmes, Barnes-Holmes, McEnteggart e Harte (2021), Barnes-Holmes, Finn et al. (2018) e Kavanagh, Barnes-Holmes, Barnes-Holmes, McEnteggart, e Finn (2018). exercidas pelos estímulos produzidos através do comportamento de relacionar, evidenciando as maneiras através das quais não só os Cfuncs são afetados pelos Crels, mas também os Crels são afetados pelos Cfuncs.

Esse foco ampliado é estruturado através da unidade conceitualizada como $R O E$ que compreende comportamentos de relacionar $(\mathrm{R})$, orientar $(\mathrm{O})$ e evocar (E). O relacionar diz respeito aos aspectos já evidenciados e discutidos pelo MDML com relação à complexidade de maneiras através das quais os seres humanos relacionam estímulos e eventos. O orientar se refere ao notar ou perceber um determinado estímulo ou evento e, por fim, o evocar diz respeito à função de um determinado estímulo ou evento, isto é, se ele é apetitivo, aversivo ou relativamente neutro (Barnes-Holmes et al., 2020).

A partir dessa ampliação, a literatura passa a se referir à estrutura MDML como HDML. Em vez de multidimensional multilevel, falamos de hyperdimensional multilevel, como uma maneira de destacar, não simplesmente a adição de novas dimensões, mas de novos focos de análise (BarnesHolmes et al., 2020).

Como implicações clínicas, a adição desses focos de análise pode orientar o olhar do terapeuta, direcionando-o e instrumentalizando-o para que possa se manter em constante análise e pensar sobre o melhor caminho para uma intervenção. $\mathrm{O}$ caminho poderia ser menos claro ou fomentando menos reflexões se nos limitássemos a dizer que o cliente está "em fusão com pensamentos sobre a sua ansiedade". As intervenções derivadas desse tipo de análise não necessariamente seriam diferentes de intervenções guiadas pelos princípios da ACT, mas a compreensão dos mecanismos subjacentes às intervenções contribui para alguns pontos importantes.

É importante ressaltar que, quando falamos de RFT, não estamos falando de uma abordagem clínica, mas de uma teoria de compreensão da linguagem. Assim, a compreensão dos princípios abordados e sua aplicação ao contexto clínico não terá sua utilidade apenas na formação de um terapeuta ACT, mas sim de qualquer terapeuta que se guia pelos princípios do Contextualismo Funcional (Villatte et al., 2015). Dessa maneira, um terapeuta que compreende como estímulos adquirem funções através da linguagem se encontra em um lugar pri- 
vilegiado para compreender e formular intervenções, ganhando assim maior flexibilidade e instrumentalização (Villatte et al., 2018).

\section{HDML e a Compreensão de Fenômenos Clínicos}

Considerando os avanços que perpassam o MDML e avançam até o modelo do HDML, é possível refletir sobre as questões iniciais que motivaram este texto sob uma nova perspectiva. Cada uma dessas perguntas pode ser explorada a partir do HDML: (1) Há no humano uma facilidade maior para o sofrimento do que para a felicidade? (2) precisaríamos recorrer ao mentalismo inerente em "confiamos mais em alguns relatos do que em outros" como causa destes fenômenos? (3) Por que relatos elogiosos ou pensamentos positivos não são o bastante?

Iniciando pela pergunta (1) há uma indagação acerca da explicação em um nível grupal, como se houvesse uma propriedade geral da espécie humana que pudesse explicar o fenômeno descrito. No entanto, o que é comum aos humanos verbalmente competentes é a capacidade de regulação verbal influenciada por diferentes níveis e dimensões. Neste sentido, se há na história de Pedro (nosso personagem fictício) um conjunto grande de estímulos verbais, coerentes entre si, que o descrevem como pouco hábil ou incapaz de superar adversidades, é muito possível que o relato de suas novas colegas seja suficiente para um estado de ansiedade exacerbada. Ademais, com alta coerência, possivelmente será evocada uma evitação da nova situação de estágio, bem como dos sentimentos evocados por essa nova situação.

Quando pensamos nos focos ampliados, trazidos pelo ROE, é possível pensar também que a rede relacional que envolve estímulos como "pouco hábil” ou "incapaz" exerce função de S- (aversiva), fazendo com que outras redes envolvidas adquiram funções de S+ (apetitivas), uma vez que funcionam para afastar o sujeito de um contexto aversivo. Um ponto interessante de se observar aqui é que o próprio "estado de ansiedade exacerbada" pode envolver um evocar com função de S+, considerando que, uma vez que o indivíduo se aproxima da ansiedade e tem seu comportamento sob controle dela (envolvendo o orientar, tal como discutido a partir do ROE), ele pode estar se afastando daquela primeira rede aversiva, o que pode contribuir para a manutenção e até potencialização dessa ansiedade. Em outras palavras, pensar, falar ou agir sobre a ansiedade se torna menos aversivo do que pensar, falar ou agir sobre eventos privados que se relacionem com os estímulos como "pouco hábil" ou "incapaz”. Assim, Pedro poderia, por exemplo, buscar uma terapia com o objetivo de tratar a ansiedade, sendo mais fácil (o que não significa agradável) lidar com ela do que com estímulos partícipes da rede relacional com função de $S$ -

Neste sentido, o impacto de um novo estímulo verbal é moderado pelas dimensões (i.e., derivação, coerência, complexidade e flexibilidade) e funções exercidas por estímulos, que foram desenvolvidas ao longo de uma história individual. Embora seja possível pensar que a evolução humana enquanto espécie possa ser responsável por uma disposição a atentar prioritariamente para estímulos aversivos, o nível ontogenético pode fornecer explicações plausíveis e úteis para a prática clínica a partir da consideração das dimensões dos repertórios verbais dos pacientes. Tais dimensões, assim como as funções que o responder em si exerce, são construídas a partir de uma história individual e são o principal desdobramento da RFT no modelo HDML.

A pergunta (2) remete precisamente ao que pode ser chamado de mentalismo enquanto uma explicação que demanda outra explicação (Baum, 2019). É razoável afirmar que confiamos mais em alguns relatos do que em outros, mas este é apenas o contexto para outras perguntas como, por exemplo, "por que confiamos mais no relato A do que no relato B?". De maneira resumida, podemos afirmar que confiamos mais no relato $\mathrm{A}$ do que no relato $\mathrm{B}$ caso A seja mais consistente com outros padrões de resposta na vida do sujeito e possua uma quantidade maior de tipos de relações coerentes envolvidas.

A pergunta (3), remete ao princípio da elaboração de procedimentos clínicos. Por que não seria suficiente, mais uma vez utilizando o exemplo fictício de Pedro, submeter seus pensamentos ansiosos resultantes da conversa com as amigas (e.g., "este estágio será demais para mim, não sou capaz de ter sucesso nesta empreitada") a uma re-estruturação? Por exemplo, ajudando Pedro a ter "pensamentos 
positivos" como "você é capaz!" ou questionando o conteúdo dos seus pensamentos ansiosos com outros pensamentos mais otimistas como "vários colegas seus já conseguiram, você também conseguirá"?

Em uma perspectiva do HDML, antes de utilizar procedimentos como estes, é necessário inicialmente uma conceitualização de caso que envolva identificar aspectos como a consistência que tais pensamentos poderiam ter com os demais pensamentos de Pedro (i.e., coerência). Quanto maior a coerência, menor a probabilidade de que o questionamento direto do conteúdo do pensamento seja uma estratégia levada a bom termo. Caso consideremos que Pedro possui um longo histórico de invalidação, por exemplo, respostas relacionadas à diminuição da sua competência possuem um longo histórico de reforçamento. Assim, da mesma forma que há uma baixa probabilidade de mudarmos nossas crenças sobre a cor do céu, ainda que alguém nos garanta que "o céu, na verdade, é rosa", Pedro dificilmente passará a acreditar que é capaz mesmo que as amigas insistam em convencê-lo com veemência e argumentação bem elaborada.

A partir dos relatos das amigas e dos autorrelatos de Pedro, a conceitualização do caso de Pedro em relação ao trabalho envolverá instâncias do relacionar (e.g., demandas do trabalho como relacionadas com "fracasso"). Tal relacionar potencialmente orientará a atenção de Pedro para todos os aspectos do trabalho que sinalizem demandas difíceis, seguido de um evocar respostas de evitação dessa situação (e.g., procurar um novo trabalho ou buscar amparo nos amigos para sua decisão de não estar neste estágio atual). Neste sentido, a reação de Pedro ao contexto do trabalho envolverá os três elementos do ROE.

É importante, também, investigar a quantidade e os tipos de relações envolvidas nos relatos e autorrelatos produzidos pela conversa com as colegas. Por exemplo, se "mais ansiedade" em função do novo desafio está em moldura de coordenação com uma história familiar de fracassos profissionais, ou de comparação com outras pessoas que seriam mais "fortes" do que ele. Isto é, qual a complexidade das relações derivadas a partir da regulação verbal produzida neste novo ambiente. Quanto mais complexas as relações que são estabelecidas como alvo, mais difícil a mudança.
Observar esta história ontogenética do Pedro também produz informações sobre a Derivação, ou seja, sobre o quanto estes novos pensamentos já possuíam lugar no repertório desta pessoa específica ou são novas unidades apenas recém-adquiridas. Quanto menor a Derivação (i.e., quanto mais a resposta já tenha sido afetada por consequências para além daquelas presentes em sua primeira emissão), menor a probabilidade de que mudanças rápidas em frequência e magnitude sejam conseguidas em um processo terapêutico, uma vez que elas já estariam sob um controle verbal e social complexo.

Em quarto e último lugar, importa observar qual a flexibilidade apresentada por Pedro em relação a mudanças nas respostas relevantes ao processo clínico. Considerando que processos de mudança clínica frequentemente envolvem relações coerentes, complexas, com baixa derivação e baixa flexibilidade, estratégias simplistas como "pensar positivo" são geralmente fadadas ao fracasso. É neste sentido que o HDML fornece o fundamento teórico e em pesquisa básica para a necessidade de refinar procedimentos clínicos capazes de lidar com cada nuance dimensional do responder relacional derivado.

\section{Conclusão}

Tais reflexões acerca do papel do HDML na compreensão de fenômenos clínicos a partir do diálogo entre a pesquisa básica e a ciência aplicada ressaltam a importância da conceitualização de caso clínico. Modelos de intervenção clínica não são meras coletâneas de procedimentos, mas envolvem uma descrição acurada dos processos psicológicos para que cada intervenção possa ser utilizada de forma contextualmente situada. Para tanto, é imprescindível que a compreensão do clínico acerca de tais processos psicológicos seja resultado de uma formação que o aproxima da investigação científica do comportamento humano e da natureza das conexões entre identificação de processos e utilização de procedimentos clínicos.

O reconhecimento desta interface entre ciência básica e aplicada para a formação do clínico tem sido reconhecida na literatura recente como um desafio para os novos modelos psicoterápicos basea- 
dos em evidências (Klepac et al., 2012). Trata-se de um desafio porque o cenário acadêmico e político em que tais modelos clínicos foram inicialmente desenvolvidos tem enfatizado, ao longo das últimas décadas, a repetição de procedimentos como a base da educação para novos psicoterapeutas (Koerner, 2013). Uma formação clínica que não é embasada na compreensão de modelos científicos translacionais capazes de fornecer explicações para o comportamento humano complexo tem gerado psicoterapeutas que não são preparados adequadamente para a prática clínica, principalmente em cenários multiculturais (Klepac et al., 2012).

O HDML é um modelo científico que visa a compreensão do comportamento humano complexo, com vistas para a fundamentação de uma prática clínica sensível às diversas nuances das contingências plurais que são típicas da clínica psicoterápica cotidiana envolvendo indivíduos verbalmente competentes. Neste sentido, é apropriado dizer que não é suficiente para o terapeuta contextual contemporâneo apropriar-se de versões da RFT sem atentar para os problemas e avanços indicados pelo HDML em sua recente sistematização.

\section{Referências}

Barnes-Holmes, D., Barnes-Holmes, Y., Luciano, C., \& McEnteggart, C. (2017). From the IRAP and REC model to a multi-dimensional multi-level framework for analyzing the dynamics of arbitrarily applicable relational responding. Journal of Contextual Behavioral Science, 6(4), 434-445. https://doi.org/10.1016/j.jcbs.2017.08.001

Barnes-Holmes, D., Barnes-Holmes, Y., \& McEnteggart, C. (2020). Updating RFT (more field than frame) and its implications for process-based therapy. The Psychological Record, 70, 605-624 https://doi.org/10.1007/s40732019-00372-3

Barnes-Holmes, Y., Barnes-Holmes, D., \& McEnteggart, C. (2018). Narrative: Its importance in modern behavior analysis and therapy. Perspectives on Behavioral Science (Special Issue on Narrative), 41(2), 509-516. https://doi. org/10.1007/s40614-018-0152-y

Barnes-Holmes, D., Barnes-Holmes, Y.,
McEnteggart, C., \& Harte, C. (2021). Back to the Future with an Up-dated Version of RFT: More Field than Frame? Perspectivas em Análise do Comportamento, 12(1). https://doi. org/10.18761/PAC.2021.v12.RFT.03

Barnes-Holmes, D., Finn, M., McEnteggart, C., \& Barnes-Holmes, Y. (2018). Derived stimulus relations and their role in a behavior-analytic account of human language and cognition. Perspectives on Behavioral Science (Special Issue on Derived Relations), 41(1), 155-173. https:// doi.org/10.1007/s40614-017-0124-7

Barnes-Holmes, Y., McEnteggart, C., \& BarnesHolmes, D. (2020): Recent Conceptual and Empirical Advances in RFT. In M. Levin, M. Twohig, \& J. Krafft (Eds.): Innovations in Acceptance and Commitment Therapy: Clinical Advancements and Applications in ACT. New Harbinger.

Baum, W. (2019). Compreender o Behaviorismo: comportamento, cultura e evolução. 3a. Ed. Artmed.

Chawla, N., \& Ostafin, B. (2007). Experiential Avoidance as a Functional Dimensional Approach to Psychopathology: An Empirical Review. Journal of Clinical Psychology, 63(9), 871-980. https://doi.org/10.1002/jclp.20400

Ferreira, T. A. S., Simões, A. S., Ferreira, A. R., \& dos Santos, B. O. S. (2020). What are values in clinical behavior analysis? Perspectives on Behavior Science, 43, 177-188. https://doi. org/10.1007/s40614-019-00219-w

Hayes, S. C. (1987). A contextual approach to therapeutic change. In N. Jacobson (Ed.), Psychotherapists in clinical practice: Cognitive and behavioral perspectives (pp. 327-387). Guilford.

Hayes, S. C., Barnes-Holmes, D., \& Roche, B. (Eds.). (2001). Relational Frame Theory: A PostSkinnerian account of human language and cognition. Plenum Press.

Hayes, S. C., Strosahl, K. D., \& Wilson, K. G. (2012). Acceptance and commitment therapy: The process and practice of mindful change. The Guilford Press.

Hughes, S., \& Barnes-Holmes, D. (2016). Relational frame theory: The basic account. In R. D. Zettle, S. C. Hayes, D. Barnes-Holmes, \& A. Biglan 
(Eds), The Wiley handbook of contextual behavioral science (pp. 129-178), Wiley-Blackwell.

Kavanagh, D., Barnes-Holmes, Y., Barnes-Holmes, D., McEnteggart, C., \& Finn, M. (2018). Exploring differential trial-type effects and the impact of a read-aloud procedure on deictic relational responding on the IRAP. The Psychological Record, 68(2), 163-176. https:// doi.org/10.1007/s40732-018-0276-1

Klepac, R. K., Ronan, G. F., Andrasik, F., Arnold, K. D., Belar, C. D., Berry, S. L., Christofff, K. A., Craighead, L. W., Dougher, M. J., Dowd, E. T., Herbert, J. D., McFarr, L. M., Rizvi, S. L., Sauer, E. M., \& Strauman, T. J. (2012). Guidelines for cognitive behavioral training within doctoral psychology programs in the United States: Report of the Inter-organizational Task Force on Cognitive and Behavioral Psychology Doctoral Education. Behavior Therapy, 43(4), 687-697. https://doi.org/10.1016/j.beth.2012.05.002

Koerner, K. (2013). What must you know and do to get good outcomes with DBT?. Behavior Therapy, 44, 568-579. https://doi.org/10.1016/j. beth.2013.03.005

Perez, W. F., Nico, Y. C., Kovac, R., Fidalgo, A. P., \& Leonardi, J. L. (2013). Introdução à Teoria das Molduras Relacionais (Relational Frame Theory): principais conceitos, achados experimentais e possibilidades de aplicação. Perspectivas em análise do comportamento, 4(1), 32-50. https://doi.org/10.18761/perspectivas. v4i1.105

Villatte, M., Villatte, J. L., \& Hayes, S. C. (2015). Mastering the clinical conversation: Language as intervention. Guilford Publications.

Villatte, M., Villatte, J. L., \& Hayes, S. C. (2018). A reticulated and progressive strategy for developing clinical applications of RFT. The Psychological Record. 68,119-121. https://doi. org/10.1007/s40732-017-0251-2

\section{Informações do Artigo}

Histórico do artigo:

Submetido em: 05/02/2021

Primeira decisão editorial: 06/04/2021

Aceito em: 13/06/2021 